\title{
Longitudinal Assessment of Progressive Retinal Pigment Epithelium Disruption in a 26 Year Old - A Multi-Spectral Imaging Case Study
}

\author{
Dawn M. Tuminello ${ }^{1}$, David T. Douglass ${ }^{2}$, Cheryl N. Zimmer ${ }^{3 *}$ and Ayda M. Shahidi ${ }^{3}$ \\ ${ }^{1}$ Consultant, Annidis Corporation, Orland Park, IL USA \\ ${ }^{2}$ Bangor, ME, USA \\ ${ }^{3}$ Annidis Corporation Ottawa, Canada
}

*Corresponding author: Cheryl N. Zimmer, Clinical Affairs and Research Manager, Annidis Corporation Ottawa, Canada, Tel: 1-855-266-4347; 807; E-mail: cherylz@annidis.com

Received date: April 29, 2016; Accepted date: July 25, 2016; Published date: July 30, 2016

Citation: Tuminello DM, Douglass DT, Zimmer CN, Shahidi AM (2016) Longitudinal Assessment of Progressive Retinal Pigment Epithelium Disruption in a 26 year old - A Multi-Spectral Imaging Case Study. J Eye Cataract Surg 2: 13. doi: 10.4172/2471-8300.100013

Copyright: (C) 2016 Tuminello DM, et al. This is an open-access article distributed under the terms of the Creative Commons Attribution License, which permits unrestricted use, distribution, and reproduction in any medium, provided the original author and source are credited.

\section{Abstract}

Background: Progressive retinal pigment epithelium (RPE) disruption in the absence of drusen or visual disturbances in young people is rarely reported in the literature, except for cases of juvenile macular degenerative diseases, pattern dystrophies or white dot syndromes. In age-related macular degeneration (AMD), RPE disruption typically does not become manifest clinically before age 55 . This case report presents a young, healthy 26 year old Caucasian male with asymptomatic progressive RPE disruption in the absence of drusen, as detected by multi-spectral imaging (MSI) technology.

Methods: A 26 year old Caucasian male was followed for progressive changes in RPE atrophy and melanin clumping over three visits in a three year period. His dilated fundus examination, fundus photography and optical coherence tomography were within normal limits. Long wavelength MSI revealed progressive RPE changes in the form of RPE atrophy and melanin clumping. MSI fundus autofluorescence (FAF) showed corresponding hyperautofluorescence in the right eye and hypoautofluorescence in the left.

Findings: Macular RPE changes can result from phototoxic effects and vary by ethnicity. Functional biomarkers to determine the risk of future vision loss with AMD are frequently sought and include complement factor $\mathrm{H}$ and Age-Related Maculopathy Susceptibility 2. FAF, for example is highly indicative of RPE dysfunction and progression. Long wavelength MSI, $620 \mathrm{~nm}$ to $740 \mathrm{~nm}$, enhances visualization of the RPE, more specifically atrophy and melanin clumping, which may be indicative of asymptomatic progressive early AMD or retinal dystrophies.

Conclusion: This case shows a longitudinal example of progressive RPE disruption in a 26 year old male. This is only one example, but multiple cases of RPE disruption in young people exist. Using MSI to further investigate RPE disruption and progression in a young population may provide a potential biomarker for early AMD, photo toxicity or other retinal dystrophies and degenerations.

\section{Abbreviations}

AMD: Age-related Macular Degeneration; APMPPE: Acute Posterior Multifocal Placoid Pigment Epitheliopathy; FAF: Fundus Autofluorescence; MEWDS: Multiple Evanescent White Dot Syndrome; MPOD: Macular Pigment Optical Density; MSI: MultiSpectral Imaging; OCT: Optical Coherence Tomography; RPE: Retinal Pigment Epithelium

\section{Introduction}

Progressive retinal pigment epithelium (RPE) disruption in the absence of drusen or visual disturbances in young people is rarely reported in the literature, except for cases of extreme macular dystrophy with associated vision loss. Juvenile macular degenerative diseases and pattern dystrophies that affect individuals in their 20's include fundus flavimaculatus, Doyne's honeycomb retinal dystrophy and Sorsby's fundus dystrophy. All include drusen as a sign in the early disease process. In the late stages of these diseases the retina resembles late age-related macular degeneration (AMD) and drusen-like lesions are less prominent [1]. Acute conditions such as multiple evanescent white dot syndrome (MEWDS) and acute posterior multifocal placoid pigment epitheliopathy (APMPPE) can be transient in nature with good visual prognoses but both are symptomatic with associated changes in vision during the active disease. MEWDS can present with subtle disruptions of the photoreceptor inner and outer segment that can be seen with optical coherence tomography (OCT). It is an inflammatory condition that presents with pathognomonic granular fovea irregularities [2]. Small, subtle RPE disturbance are not prominent in OCT scans of MEWDS. AMD is characterized by a range of degenerative changes at the macula including drusen, hyper-/hypopigmentary changes, atrophy and choroid neovascularization. RPE disruption and changes in the 
choriocapillaris typically do not become manifest clinically before the age of $55[3,4]$.

This case report presents a young healthy individual in his 20's without AMD risk factors, who shows asymptomatic progressive RPE disruption in the absence of drusen, as detected by multispectral imaging (MSI) technology.

\section{Case Report}

A 26 year old Caucasian male presented for his very first eye examination in 2013 with no ocular complaints. The patient was a non-smoker and stated that he had no known medical conditions or family history of systemic diseases. He stated that he was of Slavic descent and most family members had very minimal skin pigmentation. He denied excessive sun exposure, although spent a lot of time outside as a child. He had several skin moles but wore sunscreen to reduce his risk of melanoma. His family's ocular history was negative for eye diseases or vision loss. The patient was not taking any medications and did not have any known allergies. His best corrected visual acuities measured $20 / 20$ in each eye with refractions of plano-0.50×169 OD and $-0.25-0.50 \times 036$ OS. His pupils were equally round and reactive to light and accommodation and his irises were blue. Ocular motility testing was normal OU. The anterior segment examinations of both eyes were also unremarkable and intraocular pressures measured $17 \mathrm{mmHg}$ OU at 3:25 PM. Dilated fundus examination was unremarkable overall. A few floaters were noted in the vitreous. The fundus photographs (TRC-NW6S ${ }^{\text {TM }}$, Topcon, Japan) and Optomap ${ }^{\mathrm{TM}}$ (Optos, UK) images revealed a cup to disc ratio of $0.45 \mathrm{OU}$. Glial remnants were noted superior to the disc margin OS (Figures $1 \mathrm{~A}$ and $1 \mathrm{~B}$ ). Humphrey 24-2 automated visual field testing was done. The field OD was within normal limits. There were no indications of sensitivity loss or nerve head enlargement for the left eye (mean deviation -0.08; pattern standard deviation 1.28).
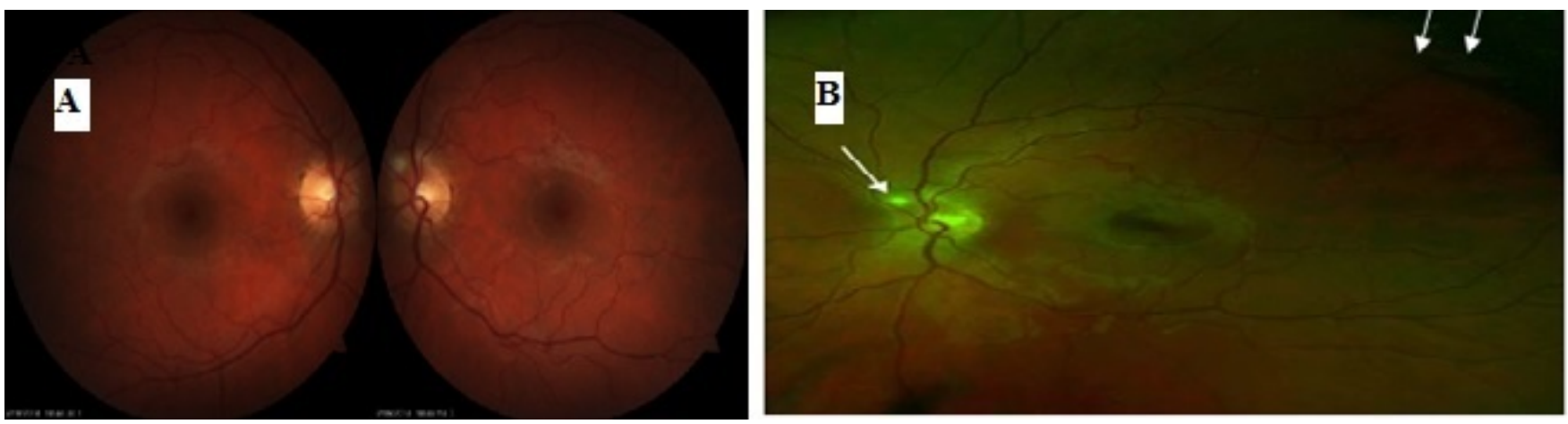

Figure 1: A) Dilated fundus photos of the patient. The glial remnants in the left eye are indicated by arrows; B): Optomap image of the patient's left eye taken in 2014. The arrow indicates the area of glial remnants.

Multi-spectral imaging of both eyes was performed using the RHA $^{\mathrm{TM}}$ (Annidis, Ottawa, Canada). The shorter wavelengths of yellow and amber (MSI-580 and MSI-590) showed no abnormalities in the fundus or the macular area and the vessels appeared healthy (Figure 2). Note that had drusen been present, they are significantly visible at this wavelength. The medium to longer wavelengths (MSI-660, MSI-690) revealed RPE changes about 2DD in size in the macular region of both eyes in the form of RPE atropy and melanin clumping (Figure 3A). The fundus autofluorescence images (MSI-FAF) showed two distinct areas of hyperautofluorescence in the right eye corresponding to the area of RPE disruption as seen in MSI-660 images Figure 4. in the same vicinity as the hyperautofluorescent area seen adjacent to the macula. At the initial visit in 2013, the patient was notifid about the RPE disruption and was advised to return to the clinic for an annual follow up exam or return sooner if experiencing any visual disturbances. He was given an Amsler grid and asked to maintain a healthy diet rich in antioxidants. The patient returned to the clinic in 2014 and 2015 and was re-imaged on the RHA to specifically look for further RPE atrophy in the macular region.
Table 1: Macula Risk PGx Laboratory Report.

\begin{tabular}{|l|l|l|}
\hline $\mathbf{2}$ Year Risk & $\mathbf{5}$ Year Risk & $\mathbf{1 0}$ Year Risk \\
\hline $3 \%$ & $8 \%$ & $17 \%$ \\
\hline 10 year Macula Risk Score & MR3 \\
\hline
\end{tabular}

Table 2: Vita Risk Pharmacogenetic Results.

\begin{tabular}{|l|l|l|l|}
\hline Gene & SNP & Genotype & Recommendation \\
\hline $\begin{array}{l}\text { Complement Factor H } \\
\text { (CFH) }\end{array}$ & rs3766405 & CC & $\begin{array}{l}\text { Low CFH and } \\
\text { Moderate ARMS2 } \\
\text { risk detected.Zinc } \\
\text { alone is } \\
\text { recommended in }\end{array}$ \\
\cline { 2 - 4 } $\begin{array}{l}\text { Age-Related } \\
\begin{array}{l}\text { Maculopathy } \\
\text { 2) }\end{array}\end{array}$ & $\begin{array}{l}372 \text { TT } 812852 \\
43 \text { ins54 } 54\end{array}$ & ND & \\
\hline
\end{tabular}

The patient was still asymptomatic and his VAs and prescription remained unchanged. The MSI-690 image of the right eye showed slight increases in size and changes in shape of the atrophic area as compared to the previous images. 


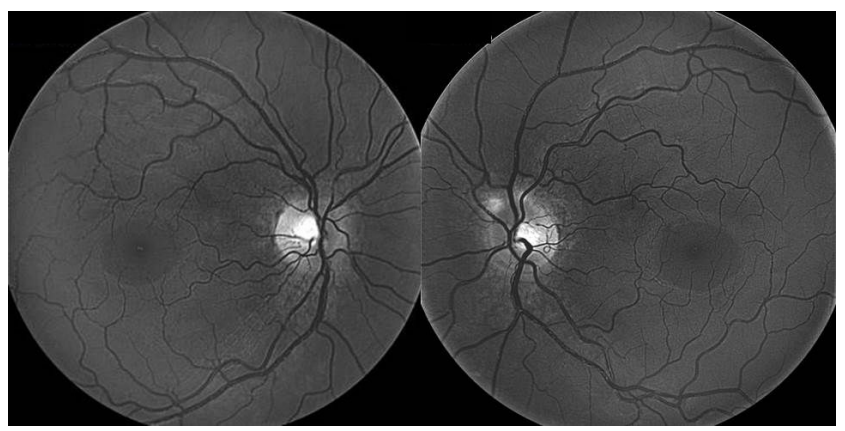

Figure 2: MSI-580 images of the patient taken at the initial visit showed a normal fundus, healthy looking vasculature and an absence of drusen.

The patient will continue to be followed yearly. The resultant is a 10 year Macula Risk Score from MR1 (low) to MR4 (high). Vita Risk Pharmacogenetics provides a biomarker based on AMD genetic risk factors. It is a suggested pharmacologic approach to management based on the patient's genotype, particularly in the CFH and ARMS2 genes. His results showed low complement factor $\mathrm{H}$ (CFH) and moderate Age-Related Maculopathy Susceptibility 2(ARMS2) (Table 2). Zinc was recommended based on this assessment. He was referred for a macula risk assessment at PDx Laboratory. He received a 10 year macula risk score of MR3 [5] with a $17 \%$ risk of progression to choroidal neovascularization or geographic atrophy (Table 1 ). The macula risk test is designed to evaluate the genetic profile of patients who are diagnosed as having early retinal changes commonly associated with AMD. An AMD risk progression profile is created using 3 components: (1) Identification of the level of AMDassociated retinal findings (2) An in office cheek swab from the patient (3) Identification of additional non-genetic risk factors commonly associated with AMD.

The patient was further imaged on the HD 5 line raster protocol of optical coherence tomography (Cirrus HD-OCT ${ }^{\mathrm{TM}}$, Carl Zeiss Meditec) where normal looking healthy retinal layers and macular thickness were observed in both eyes (Figure 6). Upon further review, while compiling this paper, one of the authors (AS) observed a minor RPE irregularity in the spectral domain OCT OD. The MSI-FAF of the left eye showed some hypoautofluorescence in the permacular area (Figure 4). MSI-940 choridal images (clinical research application only) show the underlying choroidal vasculature which appears healthy OU. Peripapillary defects are seen in the area of the glial remnants OS (Figure 5). His mascular pigment optical density (MPOD) was determined to be high with a value of 0.58 using the quantify MPS II (Zeavision, USA). A low MPOD indicates a higher risk of AMD [7].

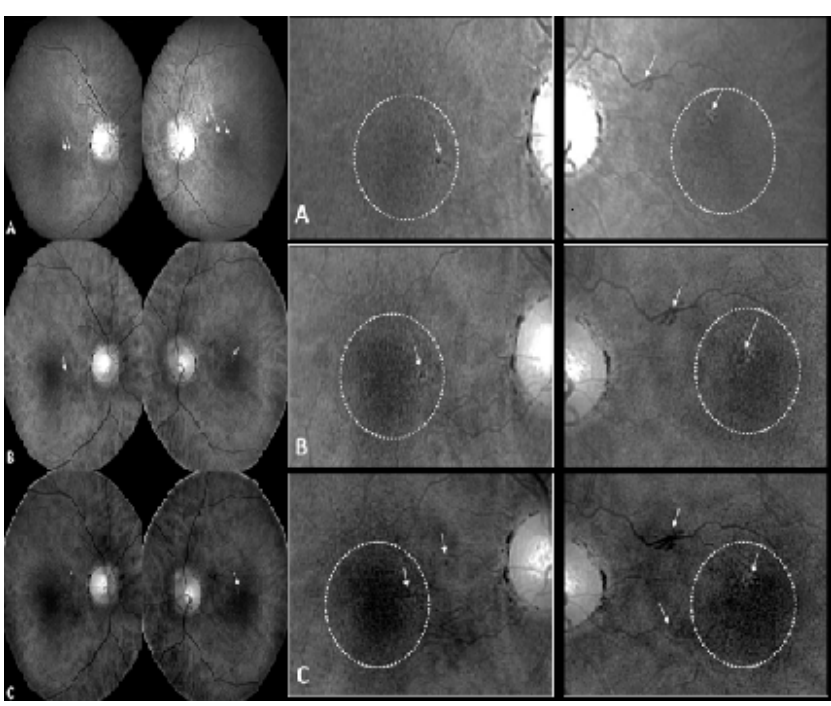

Figure 3: (LEFT) MSI-690 images of both eyes showing RPE atrophy and melanin clumping. A: At the initial visit in 2013, minor RPE changes are seen. B: Second follow up in 2014 showing enlargement of melanin clumping and RPE atrophy, as indicated by arrows. C: At the third visit in 2015, new presentations of melanin clumping were observed as shown by arrow in the right eye and the atrophy appeared to be more distinct in the left. (RIGHT) Magnified corresponding areas showing changes in the RPE.

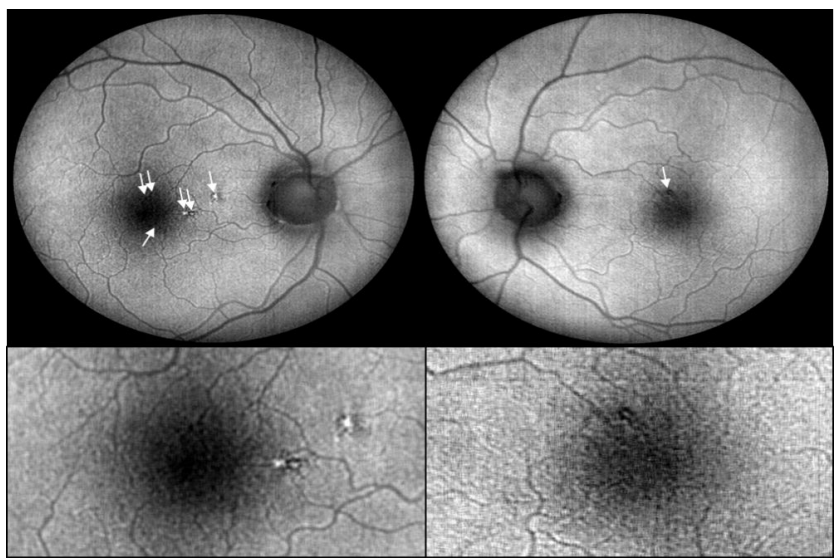

Figure 4: Two distinct areas of hyperautofluorescence in the right eye corresponding to the area of RPE disruption seen in the MSI images. The MSI-FAF of the left eye shows some hypoautofluorescence in the permacular area. 


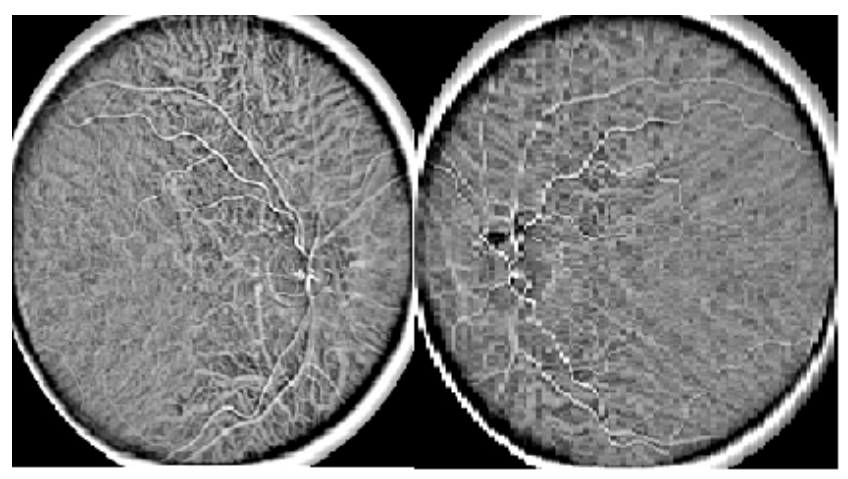

disturbances $[9,10]$. This theory has been investigated to explain the correlation between iris pigmentation and choroid melanin, macular pigment and ethnic origin [11].

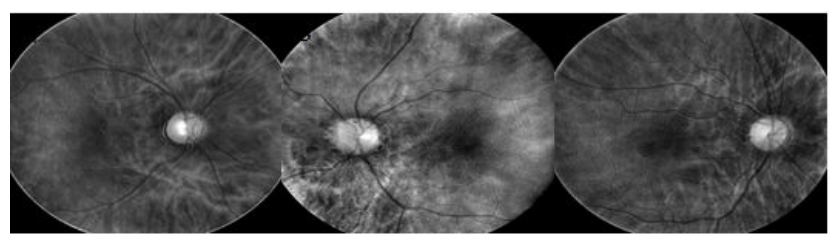

Figure 7: Sample MSI-690 images showing healthy RPEs of three Caucasians born in 1992.

Figure 5: MSI-940 choridal images (clinical research application only) show the underlying choroidal vasculature which appears healthy OU. Peripapillary defects are seen in the area of the glial remnants OS.
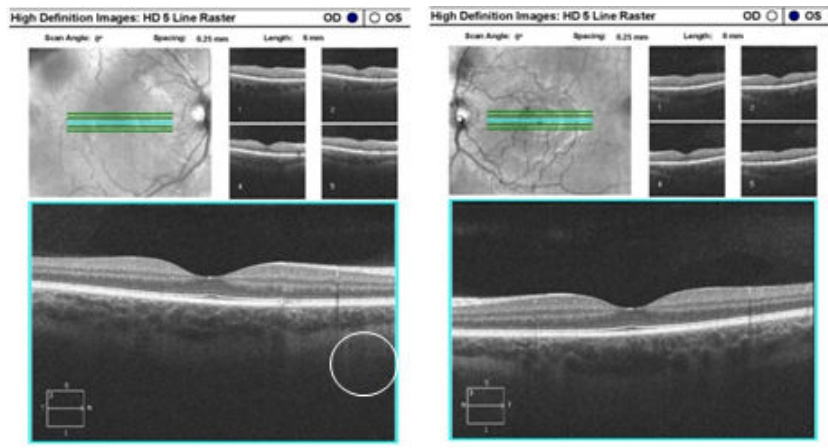

Figure 6: Spectral Domain-OCT of the patient, showing a minor RPE irregularity OD in the same vicinity as the hyperautofluorescent area seen adjacent to the macula on MSI-FAF.

\section{Discussion}

This case shows an asymptomatic progression of RPE atrophy and melanin clumping in the absence of drusen in a young healthy Caucasian over three consecutive eye examinations over a 3 year period. Generally the long wavelength MSI images of healthy Caucasians in their early to mid-twenties shows a uniform RPE with a faint macular reflex (Figure 7). The signs and symptoms of this particular patient do not fall under the categories of juvenile macular degenerative diseases, pattern dystrophies or white dot syndromes. Based on his age and health, he does not fit the category of AMD. His ethnicity and blue eyes together with the observation of RPE disruption with MSI imaging may be considered risk factors for progression to dry AMD eventually, and are definitely of concern. It is noteworthy that macular pigment depletion can occur in patients with lighter coloured irises and sun exposure. The photoreceptor/RPE complex in the outer retina is especially susceptible to phototoxic effects [8]. In animal models, near threshold light exposure has shown to be of sufficient intensity to disrupt the RPE's autofluorescence without impacting the functional ability of the outer retina or resulting in visual

Drusen and retinal pigmentary changes within the macula are well-recognized early signs of AMD [12]. The clinical importance of these features seems to vary with relation to the patient's ethnicity and racial background. AMD studies in Caucasian populations for instance, have suggested that pigmentary abnormalities are considered an early sign of AMD only when coexisting with drusen and eyes with both of these features have an increased risk of developing exudative AMD compared to eyes with drusen alone $[12,13]$. On the other hand, pigmentary abnormalities without drusen may be more important risk factors than drusen for AMD in Asian population [14]. A new clinical classification system for AMD described the relative importance of hyperpigmentary or hypopigmentary abnormalities in the presence of drusen as a significant risk factor for AMD in people over 55 years of age [15].

Retinal degeneration in AMD is hypothesized to be associated with either choriocapillaries loss as a result of RPE dysfunction and photoreceptor impairment or vice versa [16]. Regardless of the underlying cause, both hypotheses can ultimately result in loss of visual function [17]. Extensive changes in the RPE morphology have been reported in histological studies of postmortem eyes with AMD and it has been suggested that such changes can precede degeneration of overlying photoreceptors $[18,19]$.

The importance of determining the risk of future vision loss in AMD based on earlier biomarkers has been raised. SD-OCT has shown pathological changes occurring before the development of drusen-associated atrophy in cases of nascent geographic atrophy that could not be seen with fundus photography $[20,21]$. In another recent study, slowed rod-mediated dark adaptation in adults older than 60 years of age with normal macular health has been suggested to be a functional biomarker of $A M D$, where such impairment has shown to be associated with the incidence of AMD 3 years later [22].

Clinical imaging of the RPE using methods such as confocal scanning laser ophthalmoscopy (cSLO) and OCT, are useful in assessing the details of the RPE in AMD and other retinal diseases [23]. Fundus autofluorescence however provides an indication about the functional nature of the RPE. Focally increased hyperautofluorescence denotes excessive RPE fluorophores such as lipofuscin which is indicative of RPE dysfunction. These areas precede the development of atrophy in AMD [24]. 

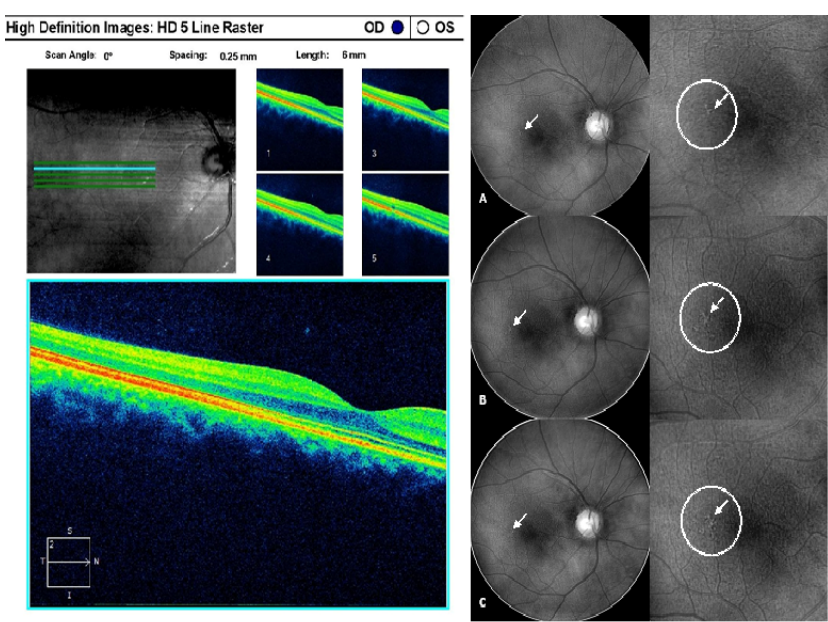

Figure 8: Example of MSI-690 images of a healthy 24 year old Caucasian female, non-smoker with no history of ocular health problems, with progressing RPE disruption in the absence of drusen. (LEFT) A: Small area of RPE atrophy is seen at the initial visit in January 2013 when she was 22 years old. B and C: Follow up visits in October 2014 and July 2016, respectively, showing melanin clumping and increased RPE atrophy. (RIGHT) Magnified corresponding areas showing successive changes in the RPE. D: Corresponding Spectral Domain-OCT 5 Line Raster of the patient in July 2016.

MSI technology brings an entirely new aspect to screening, detecting and assessing the detailed structures of the RPE. More specifically the longer wavelengths, $620 \mathrm{~nm}$ to $740 \mathrm{~nm}$, enhance visualization of the retinal pigmentation and ultimately allow the deeper retinal structures and superficial choroid to become visible by removing the effects of short wavelength scatter. This is the peak emission range for the direct observation of melanin in the RPE, which is spectrally revealed without interference from the inner retinal layers [25]. MSI shows the presence of RPE disruption, specifically melanin clumping more readily than more traditional means such as fundus photography [26]. This makes it particularly useful for detecting early RPE pathology. This case study is only one example of RPE disruption in a young person and therefore it alone does not constitute a biomarker for disease. Multiple young patients with RPE disruption in the absence of drusen have been identified by the authors and others using MSI (Figure 8). This case and others like it are the basis for larger, longitudinal clinical studies of RPE disruption in young people in the absence of drusen. RPE disruption may be a possible recognized biomarker of disease in the future, be it $A M D$, photo toxicity or the earliest manifestation of another form of macular dystrophy or degeneration, not readily found due to visualization and imaging limitations. It is possible that the type of RPE disruption illustrated here is very early and only asymptomatic for the time being. Continued progression to symptomology is possible. Multi-spectral imaging is the ideal instrument to follow such cases.

\section{Conflict of Interest}

Dawn M. Tuminello is a consultant for the Annidis Clinical Interpretation Center, Cheryl N. Zimmer is the Clinical Affairs and Research Manager at Annidis Corporation and Ayda M. Shahidi is the Research Manager at Annidis Corporation.

\section{References}

1. Alkuraya H, Zhang K (2010) Pattern Dystrophy of the Retinal Pigment Epithelium. Retinal Physician.

2. Nguyen MHT, Witkin AJ, Reichel E, Ko TH, Fujimoto JG, et al. (2007) Microstructural Abnormalities in MEWDS Demonstrated by Ultrahigh Resolution Optical Coherence Tomography. Retina 27: 414-418.

3. Klein R, Peto T, Bird A, Vannewkirk MR (2004) The epidemiology of age-related macular degeneration. Am J Ophthalmol 137:486495.

4. Beisekeeva J, Gorgels TG, Bergen AA, Booji JC, Baas DC (2010) The dynamic nature of Bruch's membrane. Prog Retin Eye Res 29:1-18.

5. Seddon JM, Sharma S, Adelman RA (2006) Evaluation of the clinical age-related maculopathy staging system. Ophthalmology 113: 260-266.

6. Awh CC, Lane AM, Hawken S, Zanke B, Kin IK (2013) CFH and ARMS2 genetic polymorphisms predict response to antioxidants and zinc in patients with Age-related Macular Degeneration. Ophthalmology 120: 2317-2323.

7. Van Der Veen RL, Berendschot TT, Hendrikse F, Carden D, Murray IJ, et al. (2009) A new desktop instrument for measuring macular pigment optical density based on a novel technique for setting flicker thresholds. Ophthal Physiol Opt 29: 127-137.

8. Hunter JJ, Morgan JI, Merigan WH, Sliney DH, Sparrow JR, et a. (2012) The susceptibility of the retina to photochemical damage from visible light. Prog Retin Eye Res 31: 28-42.

9. Masella BD, Hunter JJ, Williams DR (2014) Rod Photopigment Kinetics after Photodisruption of the Retinal Pigment Epithelium. Invest Ophthalmol Vis Sci 55: 7535-7544.

10. Arnault E, Barrau C, Nanteau C, Gondouin P, Bigot K, et al. (2013) Phototoxic Action Spectrum on a Retinal Pigment Epithelium Model of Age-Related Macular Degeneration Exposed to Sunlight Normalized Conditions. PLoS ONE 8: e71398.

11. Hammond BR, Fuld K, Snodderly DM (1996) Iris color and macular pigment optical density. Exp Eye Res 1996;62: 293-297.

12. Klein R, Klein BE, Tomany SC, Meuer SM, Huang GH (2002)Tenyear incidence and progression of age-related maculopathy: The Beaver Dam eye study. Ophthalmology 109: 1767-1779.

13. Wang JJ, Foran S, Smith W, Mitchell P (2003) Risk of age-related macular degeneration in eyes with macular drusen or hyperpigmentation: the Blue Mountains Eye Study cohort. Arch Ophthalmol. 121: 658-663.

14. Sasaki M, Kawasaki R, Uchida A, Koto T,Shinoda H, et al. (2014) Early Signs of Exudative Age-Related Macular Degeneration in Asians. Optom Vis Sci 91: 849-853.

15. Ferris FL, Wilkinson CP, Bird A, Chakravarthy U, Chew E, et al. (2013) Clinical classification of age-related macular degeneration. Beckman Initiative for Macular Research Classification Committee Ophthalmology 120: 844-51. 
16. McLeod DS, Grebe R, Bhutto I, Merges C, Baba T, et al. (2009) Relationship between RPE and Choriocapillaris in Age-Related Macular Degeneration. Invest Ophthalmol Vis Sci50: 4982-4991.

17. Katz ML (2002) Potential role of retinal pigment epithelial lipofuscin accumulation in age-related macular degeneration. Arch Gerontol Geriatr 34: 359-70.

18. Rudolf M, Vogt SD, Curcio CA, Huisingh C, McGwin G, et al. (2013) Histologic basis of variations in retinal pigment epithelium autofluorescence in eyes with geographic atrophy. Ophthalmology. 120: 821-828.

19. Adler R, Curcio C, Hicks D, Price D, Wong F (1999) Cell death in age-related macular degeneration. Mol Vis 5: 31.

20. Wu Z, Luu CD, Ayton LN, Goh JK, Lucci LM, et al. (2014) Optical Coherence Tomography-Defined Changes Preceding the Development of Drusen-Associated Atrophy in Age-Related Macular Degeneration. Ophthalmology 121: 2415-2422.

21. Wu Z, Luu CD, Ayton LN, Goh JK, Lucci LM, et al. (2015) Fundus Autofluorescence Characteristics of Nascent Geographic Atrophy in Age-Related Macular Degeneration. Invest Ophthalmol Vis Sci 56: 1546-1552.
22. Owsley C, McGwin G, Clark ME, Jackson GR, Callahan MA, et al. (2016) Delayed Rod-Mediated Dark Adaptation Is a Functional Biomarker for Incident Early Age-Related Macular Degeneration. Ophthalmology 123: 344-351.

23. Schmitz-Valckenberg S, Fleckenstein M, Gobel AP, Sehmi K, Fitzke $\mathrm{FW}$, et al. (2008) Evaluation of autofluorescence imaging with the scanning laser ophthalmoscope and the fundus camera in agerelated geographic atrophy. Am J Ophthalmol 146: 183-192.

24. Holz FG, Bellman C, Staudt S, Schütt F, Völcker HE (2001) Fundus autofluorescence and development of geographic atrophy in agerelated macular degeneration. Invest Ophthalmol Vis Sci 42: 1051-1056.

25. Zimmer C, Kahn D, Clayton R, Dugel P, Freund KB (2014) Innovation in diagnostic retinal imaging: multispectral imaging. Retina Today 9: 94-99.

26. Dugel PU, Zimmer CZ (2016) Imaging of Melanin Disruption in Age-Related Macular Degeneration Using Multispectral Imaging. OSLI Retina 47: 134-141. 